http://jmscr.igmpublication.org/home/ ISSN (e)-2347-176x ISSN (p) 2455-0450 crossref DOI: https://dx.doi.org/10.18535/jmscr/v8i1.53

\title{
Performance of Low Dose Non Enhanced Multidetector CT in Evaluation of Urinary Calculi
}

Authors

\author{
Dr P. Sirisha Naidu ${ }^{1}$, Dr Sonali Ullal ${ }^{2}$, Dr Ajit Mahale ${ }^{3}$, Dr Ashvini Kumar ${ }^{4}$ \\ ${ }^{1}$ Senior Resident, ${ }^{2}$ Associate Professor, KMC, MAHE, ${ }^{3,4}$ Professor, KMC, MAHE \\ *Corresponding Author \\ Dr Sonali Ullal \\ Department of Radiodiagnosis, Kasturba Medical College, Mangalore, \\ Manipal Academy of Higher Education
}

\begin{abstract}
Nephrolithiasis is a chronic recurrent problem and it is increasing in incidence in younger generation, patients are getting exposed repetitively to $C T$ (Computed tomography) radiation with its attendant radiation hazards. Hence CT dose reduction technique happens to be the need of the hour to reduce the dose burden to the patient. So it is necessary to tailor a new and finer protocol with reduced dose without compromising optimum image quality.

Aims: To assess the efficiency of low dose MDCT (Multidetector Computed tomography) in detecting urinary calculus when compared to standard MDCT.

Methods and Material: This is a prospective cross sectional study of 52 adult patients, carried on Multidetector 16 slice CT scanner-G E Bright speed elite over a period of 18 months from November 2016 to May 2018. All the patients who were advised CT urogram or CT KUB in whom urinary calculus was detected by MDCT-KUB (kidney, ureter and bladder) using an average tube potential of $120 \mathrm{kVp}$ and tube current of 270- $350 \mathrm{mAs}$ with a slice thickness of $5 \mathrm{~mm}$ were selected and these patients were resubjected to low dose CT at the level of urinary calculus by using $100 \mathrm{kVp}$ and $100 \mathrm{mAs}$ with a slice thickness of $5 \mathrm{~mm}$ and reconstructed at $1.25 \mathrm{~mm}$.

Results: The mean stone size in standard dose is $13.395 \mathrm{~mm}$ and in low dose is $13.361 \mathrm{~mm}$ with no significant difference.

Mean CTDivol (Computed tomography dose index) in standard and low dose are respectively are 13.876 $m G y$ and $2.796 m G y$ with average dose reduction by $79.92 \%$ in low dose.

Conclusions: Low dose CT was found to be equally sensitive with equal positive predictive value in the detection and localisation of stones when compared to standard dose CT. At the same time low dose protocol helped to reduce patient radiation dose by an average of $79.92 \%$. Since urolithiasis is a recurring disease and patients are frequently subjected to repeat CT examinations, the present low dose CT protocol will help to reduce cumulative radiation dose to the patient without compromising the sensitivity.

Keywords: CT dose index, Low dose CT, radiation dose, renal calculus, urolithiasis.
\end{abstract}

\section{Introduction}

Renal calculus disease is a common clinical ailment encountered in daily practice in both sexes of all age groups, most common in middle age people. It has a high recurrence rate $^{1}$ with an increasing trend towards affecting the younger generation ${ }^{2}$. It is more common in males ${ }^{3}$. 
Its incidence is increasing in both developed and developing countries due to changes in socioeconomic conditions.

Various imaging modalities are currently available for evaluation of urolithiasis

\section{X-Ray KUB 2.Ultrasound 3 Intravenous Urography 4.MDCT (Multidetector Computed tomography)}

X-Ray KUB- has reduced sensitivity (45-58\%) and specificity (69-77\%) ${ }^{4}$ because of overlying bowel gas, radiolucent stones, extrarenal and extra ureteral calcifications.

Ultrasound- has its own limitation in case of morbidly obese patients and gaseous abdomen. It is more of operator dependent investigation.

X- RAY KUB with Ultrasound- sensitivity $96 \%$ specificity $51 \%{ }^{4}$

IVU-has sensitivity $85.2 \%$ specificity $90.4 \%{ }^{4}$.Its limitations are

- Missing small stones.

- Edema or swelling at the vesicoureteric junction after the passage of stones mimicking the appearance of a retained stone.

- Risks associated with intravenous contrast.

- The study might be limitation by inadequate bowel preparation, bowel ileus, swallowed air and technical variability.

- Inconvenience of a long filming sequence.

MDCT-Currently non-contrast CT (NCCT) is considered first-line imaging study ${ }^{4}$ for evaluation of patients with acute flank pain and suspected stone because it overcomes the above limitations. It is sensitive even in cases of small radiolucent stones and even in obese patients.

Sensitivity-95 to $98 \%{ }^{4}$

Specificity-96 to $98 \%{ }^{4}$

CT KUB is recognised as the best clinical imaging modality for patients with suspected urolithiasis as per the ACR (America College of radiology) Appropriateness Criteria. ${ }^{5}$

As low as reasonably achievable (ALARA) principle should be followed while making dose reduction and unnecessary radiation exposure.
Effective radiation dose can be optimized by decreasing tube potential, automatic current modulation, and CT post processing. ${ }^{6}$

- Pierre-Alexandre Poletti et al (2007) evaluated one hundred and twenty-five patients ( 87 men, 38 women; mean age, 45 years) who were admitted with suspected renal colic and underwent both abdominal low-dose CT at $30 \mathrm{mAs}$ and standard-dose $\mathrm{CT}$ at $180 \mathrm{mAs}$.

In patients with a BMI of $<30$, low-dose CT achieved a sensitivity of $95 \%$ and a specificity of $97 \%$ for detecting ureteric calculi. In patients with a BMI of $<30$, lowdose CT was $86 \%$ sensitive for detecting ureteric calculi of $<3 \mathrm{~mm}$ and $100 \%$ sensitive for detecting calculi of $>3 \mathrm{~mm}$. Low-dose CT was $100 \%$ sensitive and specific for depicting non -urinary tract related disorders ${ }^{7}$.

- William Sohn et al (2013) performed low dose CT (50-150mA) versus standard dose CT(200-500mA) for determination of stone size, density and skin to stone distance with results showing no significant difference but with effective radiation dose reduction from 23 to $6 \mathrm{mSv}$ (73\% dose reduction) ${ }^{8}$.

So the present study is aimed to assess the efficiency of low dose MDCT in detecting urinary calculus when compared to standard MDCT and to evaluate

a. Sensitivity and specificity of low dose MDCT in detecting and localisation of urinary calculus.

b. Comparison of mean Hounsfield units and correlating with biochemical report wherever possible.

c. Assessing CTDi volume (Computed tomography dose index) and percentage dose reduction.

\section{Subjects and Methods}

- This is a prospective cross sectional study of 52 adult patients carried on Multidetector 16 slice CT scanner-G E Bright speed elite over a period of 18 
months November 2016 to May 2018 in Department of Radiodiagnosis, Kasturba medical college, Ambedkar circle, Mangalore, Manipal academy of higher education (MAHE).

- Assuming Sensitivity $-97 \%$ \&Specificity$95 \%{ }^{1}$ with confidence interval $95 \%$ and power $90 \%$.

- SAMPLE SIZE $n=52$ based on formula $\mathrm{n}=\mathrm{Z} \alpha 2 \times \operatorname{Sn}(1-\mathrm{Sn})$

\section{. L2 $\mathrm{LP}$ \\ [Z $\alpha: 1.96$ L : Error P : Prevalence]}

- Statistical analysis was done by kappa statistics -sensitivity and positive predictive value.

- All the patients who were advised CT urogram or CT KUB in whom urinary calculus was detected by MDCT-KUB using an average tube potential of $120 \mathrm{kVp}$ and tube current of 270- $350 \mathrm{mAs}$ with a slice thickness of $5 \mathrm{~mm}$ were selected and these patients were resubjected to low dose CT at the level of urinary calculus by using 100 $\mathrm{kVp}$ and $100 \mathrm{mAs}$ with a slice thickness of 5 $\mathrm{mm}$ and the images are later reconstructed at $1.25 \mathrm{~mm}$.

Standard MDCT KUB protocol in the department:

- Tube voltage $120 \mathrm{kVp}$

- Tube current 270-350 mAs

- Slice thickness $5 \mathrm{~mm}$

- Reconstruction $1.25 \mathrm{~mm}$

LOW DOSE CT protocol in my present study:

- Tube voltage $100 \mathrm{kVp}$ for average BMI

- Tube current $100 \mathrm{mAs}$

- Slice thickness $5 \mathrm{~mm}$

- Reconstruction $1.25 \mathrm{~mm}$

\section{Inclusion and Exclusion Criteria Inclusion criteria}

- All patients with suspected renal colic who were advised CT urogram or CT KUB and the initial plain CT has showed urinary calculus .

\section{Exclusion criteria}

- Pregnancy

- Children

- $\quad \mathrm{BMI} \geq 30 \mathrm{~kg} / \mathrm{m}^{2}$

\section{Results}

Our study included 36 are male and 16 are female with male:female ratio-2:1

Our study included patients of age group ranging from 19 to 78 years with mean age group 47 years.

In our study based on location the highest percentage of stones are found in kidney followed by ureter.

The mean stone size in high dose is $13.395 \mathrm{~mm}$ and in low dose is $13.361 \mathrm{~mm}$ with no significant difference.

Mean density of stone in standard dose CT and in low dose CT is 876.5 and $905.1 \mathrm{HU}$ respectively with no significant difference.

Mean CTDivol in high and low dose are respectively are $13.876 \mathrm{mGy}$ and $2.796 \mathrm{mGy}$ with average dose reduction by $79.92 \%$ in low dose.

Out of 52 cases, stone retrieval was done for 20 cases for which follow up was done

Most of the stones were mixed stones with composition of calcium, oxalate, ammonia and uric acid.

We came across one pure uric acid stone which shows a density of $311 \mathrm{HU}$.

Out of the 20 cases Calcium containing stones were 16 with density ranging from $333 \mathrm{HU}$ to $1565 \mathrm{HU}$, which included staghorn, pelvic, ureteric and VUJ calculi .

Three mixed stones of uric acid and oxalate composition of density ranging from 634 to 1455 HU were found.

Density of stones helps us to determine the calculi which respond to extracorporeal shock wave lithotripsy, stones that are likely to fragment easily from those that would require a greater number of shock waves. Lesser the density of calculus, easier to fragment by extracorporeal shock wave lithotripsy ${ }^{9}$. 


\section{JMSCR Vol||08||Issue||01||Page 355-361||January}

Male patients -36 Female patients- 16

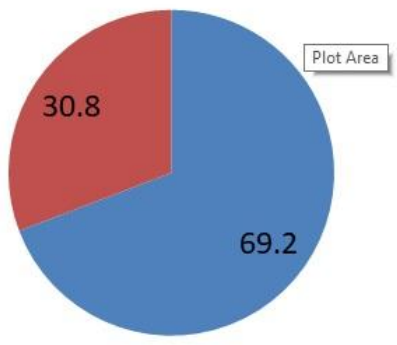

MALE

FEMALE

Figure 1 Pie Chart Representing Sex Distribution of the Patients

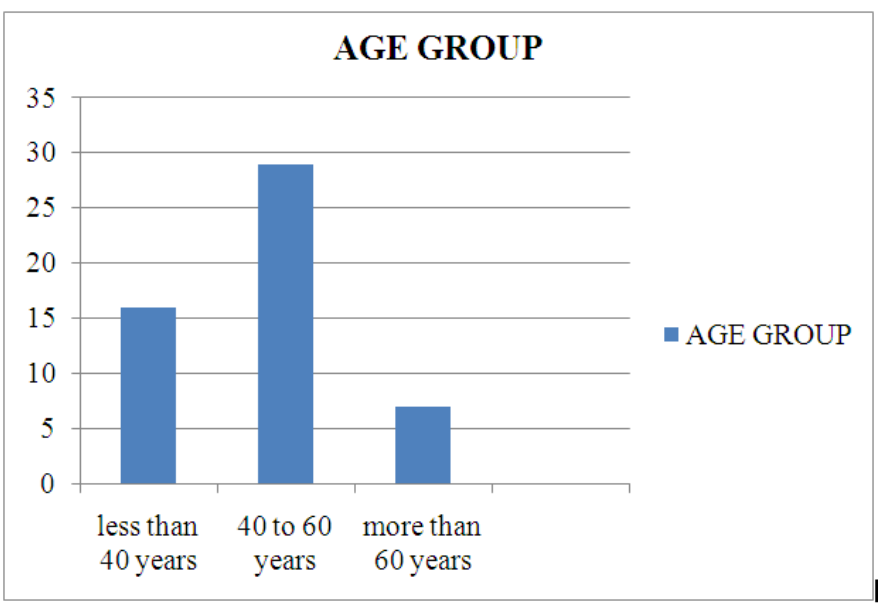

Table 1 Age Distribution of the Patients

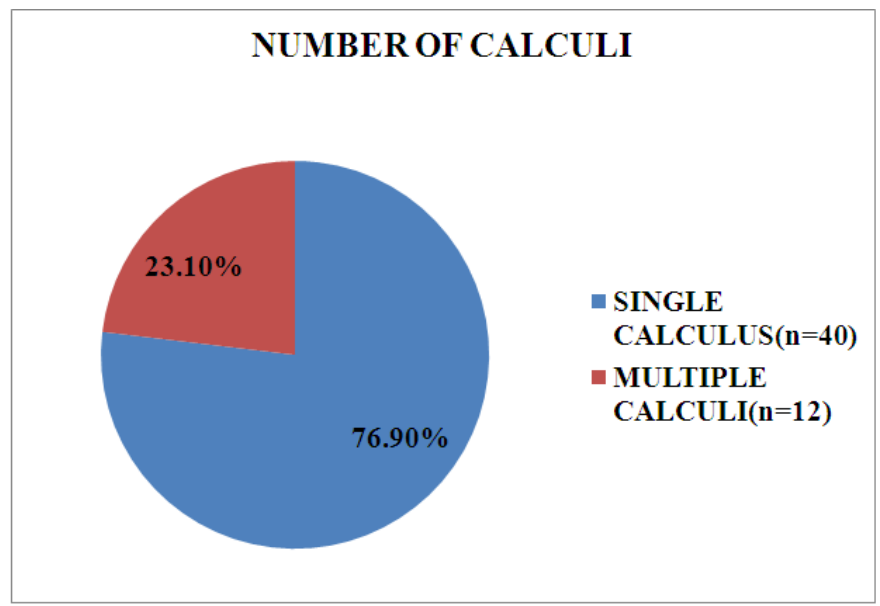

Figure 2 Representing the ratio of Patients with single and Multiple Calculi in the Study Group
LOCATION

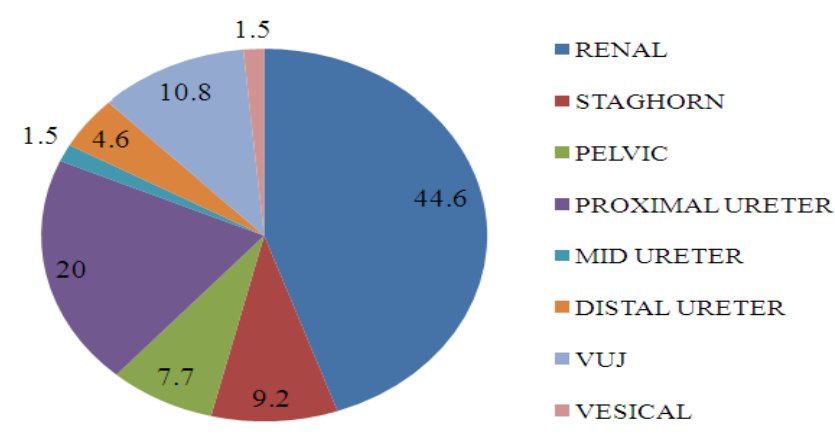

Figure 3 Representing the Location of Stone

Table 2 Represents Mean, Median, and Range of Calculus in Standard and Low Dose Protocols.

\begin{tabular}{|c|c|c|c|c|c|c|}
\hline & $\begin{array}{l}\text { SIZE } \\
(\mathbf{m m})\end{array}$ & $\begin{array}{l}\text { SIZE } \\
(\mathbf{m m})\end{array}$ & $\begin{array}{c}\text { DENSITY } \\
(\mathrm{HU})\end{array}$ & $\begin{array}{c}\text { DENSI } \\
\text { TY } \\
\text { (HU) } \\
\end{array}$ & $\begin{array}{c}\text { CTDi vol } \\
(\text { (mGy) }\end{array}$ & $\begin{array}{c}\text { CTDi vol } \\
\text { (mGy) }\end{array}$ \\
\hline & SD & LD & SD & LD & SD & LD \\
\hline N Valid & 64 & 64 & 64 & 64 & 52 & 52 \\
\hline Mean & 13.395 & 13.361 & 876.453 & 905.109 & 13.876 & 2.796 \\
\hline Median & 9.450 & 9.800 & 885.500 & 943.500 & 13.800 & 3.000 \\
\hline Range & 56.300 & 56.000 & 1316.000 & $\begin{array}{c}1406.00 \\
0\end{array}$ & 7.180 & 1.200 \\
\hline Minimum & 3.00 & 3.000 & 185.000 & 227.000 & 10.090 & 1.800 \\
\hline Maximum & 59.000 & 59.000 & 1501.000 & $\begin{array}{c}1633.00 \\
0\end{array}$ & 17.270 & 3.000 \\
\hline
\end{tabular}

SD-Standard Dose

LD Low Dose

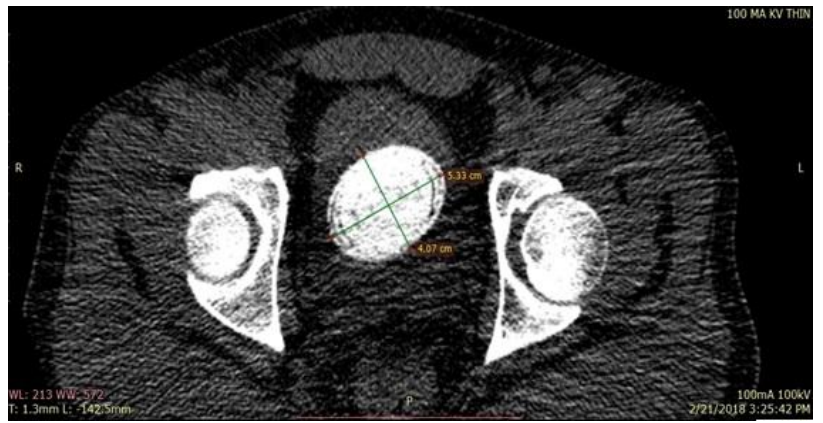

Fig 4.1 Low dose CT - Laminated vesical calculus measuring $53 \times 40 \mathrm{~mm}$

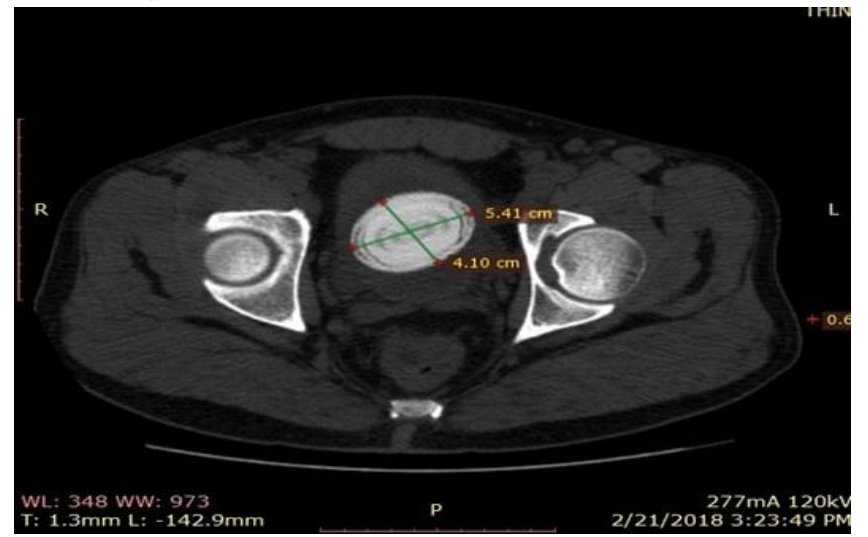

Fig 4.2 Standard dose CT -Laminated vesical calculus measuring $54 \times 41 \mathrm{~mm}$ 


\section{JMSCR Vol||08||Issue||01||Page 355-361||January}

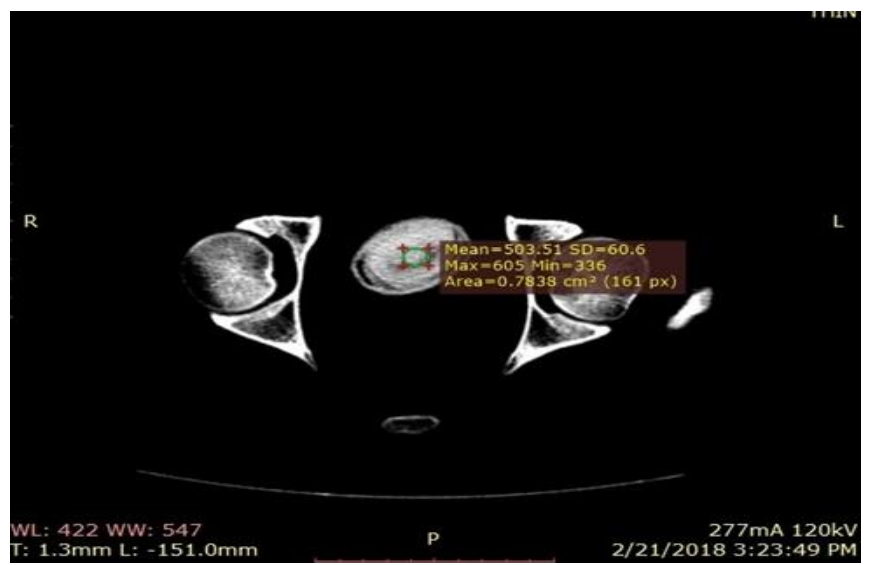

Fig 4.3 Standard dose CT -Mean density of vesical calculus is $503 \mathrm{HU}$

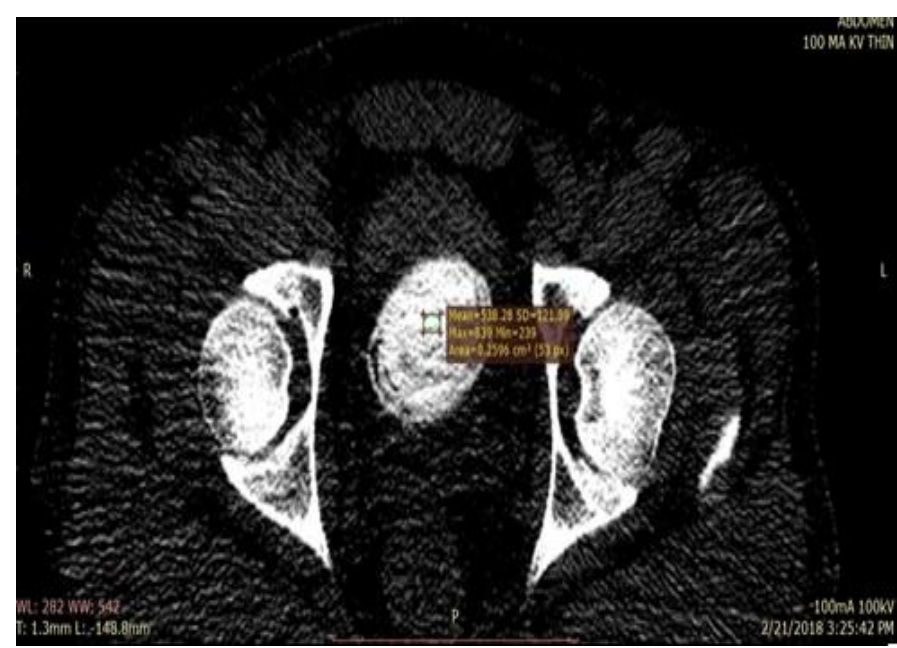

Fig 4.4 Low dose CT - Mean density of the vesical calculus is $538 \mathrm{HU}$

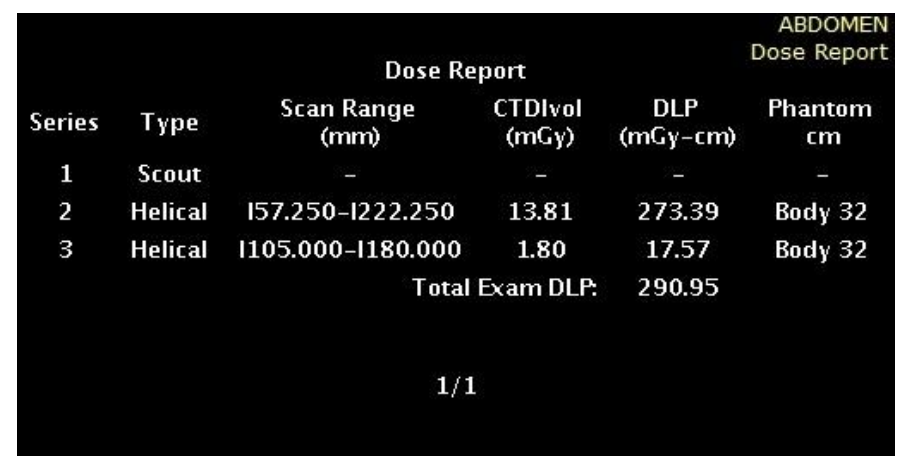

Fig 4.5 CTDIvol of standard dose CT is $13.81 \mathrm{mGy}$ and of low dose CT is $1.8 \mathrm{mGy}$ respectively which is $86 \%$ dose reduction

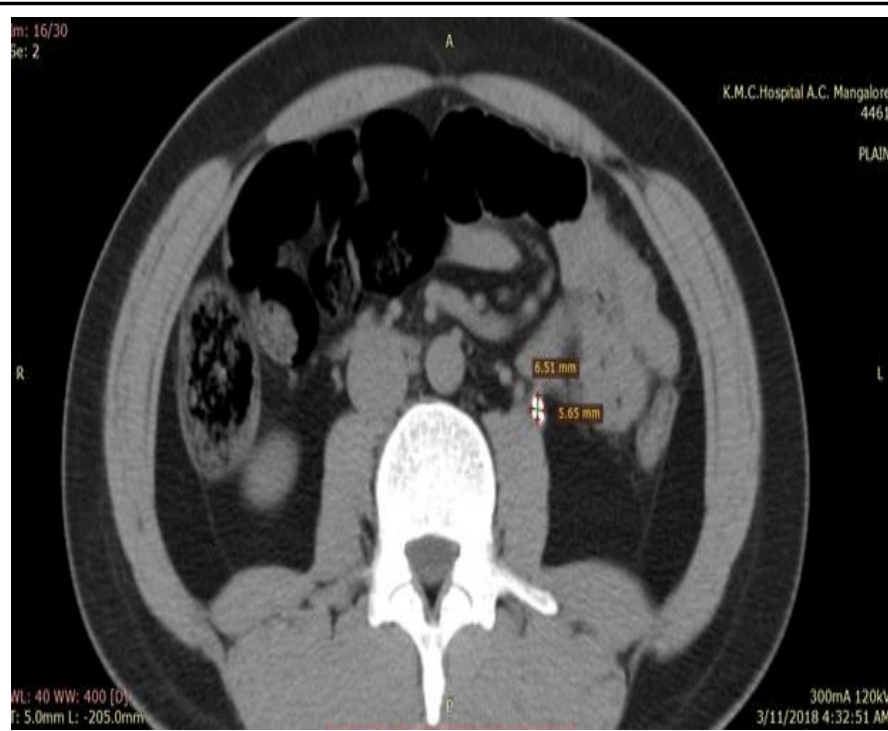

Fig 5.1 Standard dose CT - Left proximal ureteric calculus measuring $6.5 \times 5.6 \mathrm{~mm}$

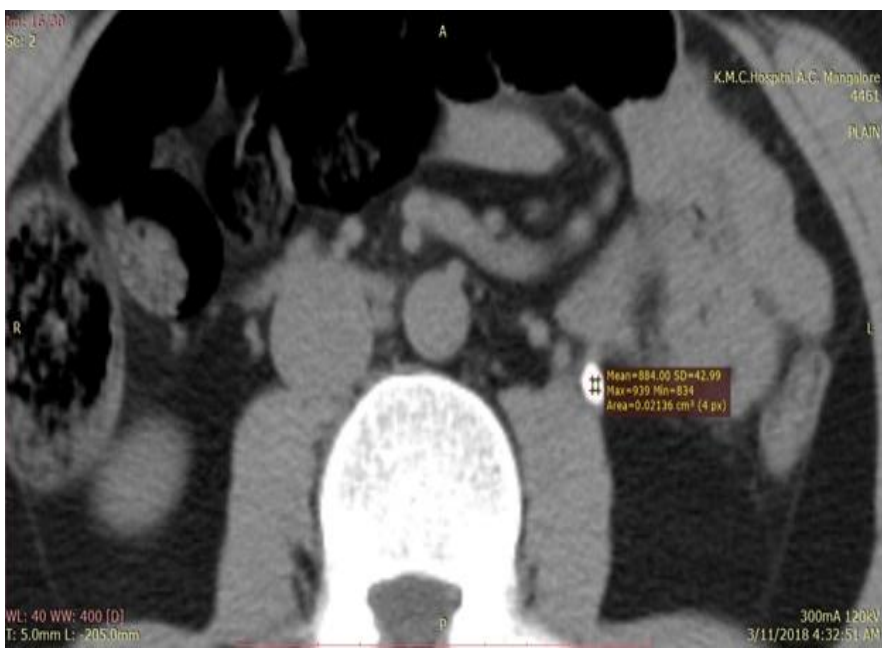

Fig 5.3 Standard dose CT - Left proximal ureteric calculus of density $884 \mathrm{HU}$

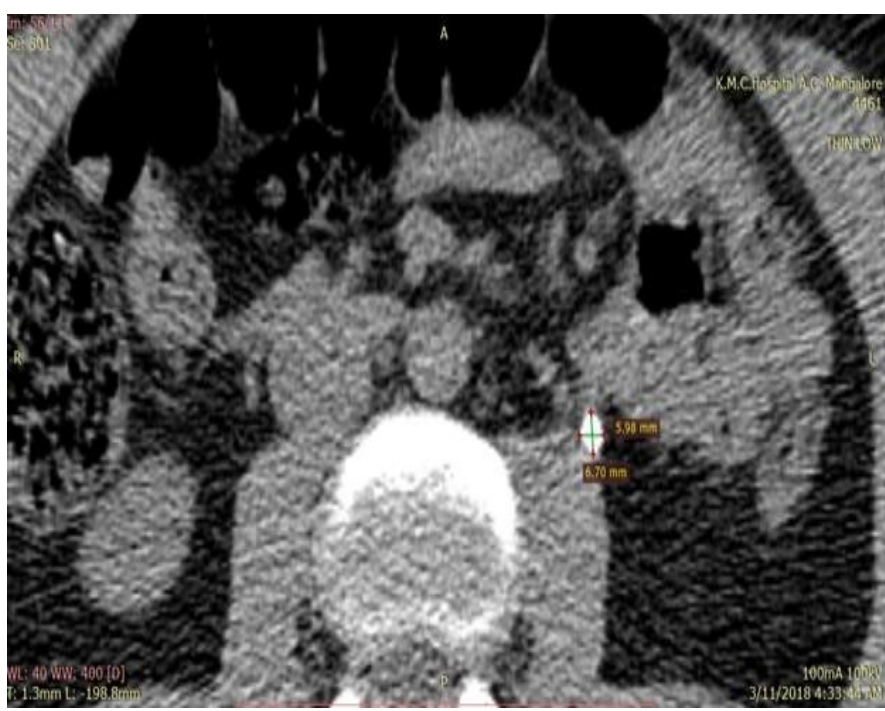

Fig 5.2 Low dose CT - Left proximal ureteric calculus measuring $6 \times 6.7 \mathrm{~mm}$ 


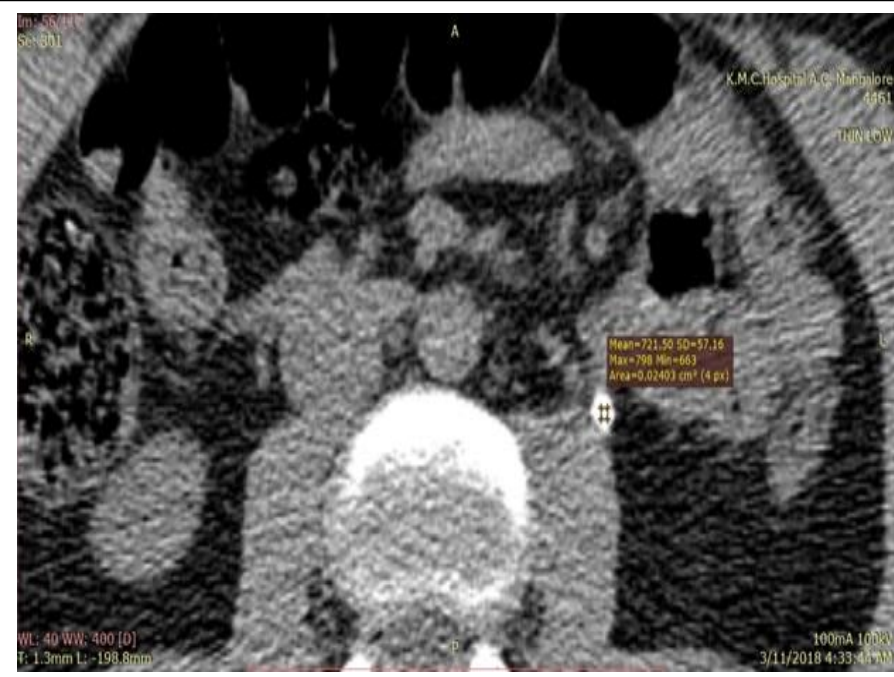

Fig 5.4 Low dose CT - Left proximal ureteric calculus of density $721 \mathrm{HU}$

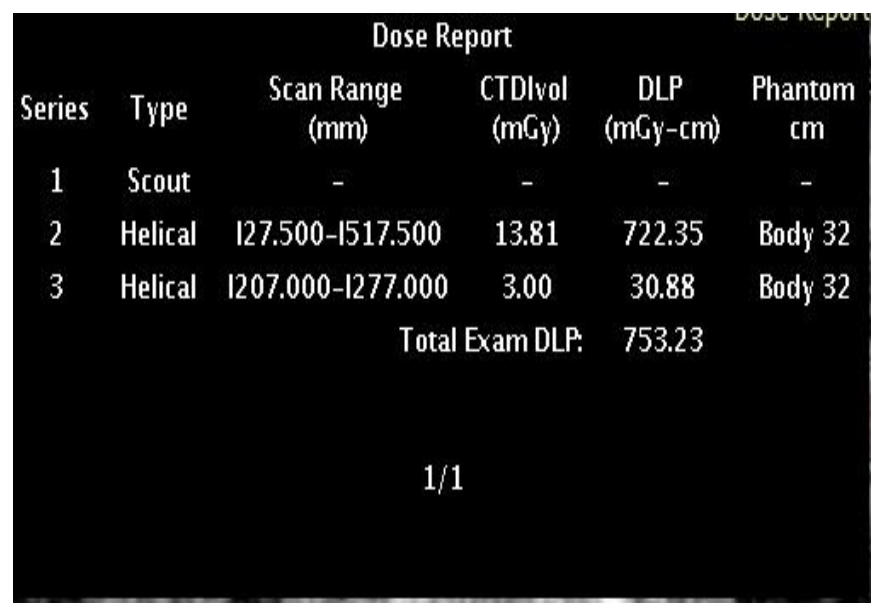

Fig 5.5 CTDIvol of standard dose CT is $13.8 \mathrm{mGy}$ and of low dose CT is $3 \mathrm{mGy}$ respectively which is $78 \%$ dose reduction

\section{Discussion}

The present study will help us to develop a low dose CT protocol for renal calculus disease while maintaining the diagnostic quality of the image with effective dose reduction to the patient.

We can manipulate various technical factors during CT acquisition to bring a substantial reduction in radiation dose. The relationship between the tube current and radiation dose is proportional which implies that reduction of mAs by $50 \%$ will result in reduction of radiation dose by $50 \%$. Radiation dose is directly proportional to $\mathrm{kV} 2$, so significant reduction in dose can be achieved with relatively small decrements in tube potential. Altering slice thickness and pitch can also result in dose reduction.
In the present study, I have reduced tube current as well as tube potential for dose reduction from 270$350 \mathrm{mAs}$ to $100 \mathrm{mAs}$ and from $120 \mathrm{kVp}$ to $100 \mathrm{kVp}$ respectively.

Standard MDCT KUB protocol in the department:
- Tube voltage $120 \mathrm{kVp}$
- Tube current 270-350 mAs
- Slice thickness $5 \mathrm{~mm}$
- Reconstruction $1.25 \mathrm{~mm}$

LOW DOSE CT protocol in my present study:

- Tube voltage $100 \mathrm{kVp}$ for average BMI

- Tube current $100 \mathrm{mAs}$

- Slice thickness $5 \mathrm{~mm}$

- Reconstruction $1.25 \mathrm{~mm}$

In our present study, the CTDI using low dose CT protocol was found to be much lower when compared to that of standard dose CT protocol.

\section{Radiation Dose Calculation}

$\mathrm{E}=\mathrm{DLP} \times \mathrm{k}$ Where $\mathrm{E}=$ Effective Dose in $\mathrm{mSv}$, DLP $=$ Dose Length Product in mGyxcm

$\mathrm{k}=$ conversion coefficient in $\mathrm{mSv} / \mathrm{mGyxcm}=.015$ for abdomen 10

CTDI vol= Approximates the average dose in the acrylic dosimetric phantom during a helical CT scan that covers the entire phantom.

Jiang Licheng et $\mathrm{al}^{10}$ compared unenhanced lowdose spiral CT localization with unenhanced standard-dose spiral CT in patients with upper urinary tract calculi for minimally invasive percutaneous nephrolithotomy (MPCNL) treatment. They performed standard and low dose CT protocol with following parameters $120 \mathrm{kV}, 100 \mathrm{mAs}$ and $120 \mathrm{kV} ; 25 \mathrm{mAs}$ respectively .

They determined the size and location of calculi in the urinary system and the volume CT dose index (CTDIvol) and scan length (L) The effective dose (E) of CT scan was obtained by the following formula: $\mathrm{E}=\mathrm{CTDIvol} \times \mathrm{L} \times \mathrm{f}$ (where $\mathrm{f}$ is a specific conversion factor, and for which the $\mathrm{f}$ of abdominal region is 0.015 ) in both low and standard dose CT.

Effective dose $(\mathrm{mSv})$ of low dose CT protocol is $0.88 \pm 0.10$ where as for standard protocol it is 3.58 \pm 0.38 .They found low-dose CT was 100 percent sensitive and 100 percent specific for depicting the 
location of the renal and ureteric calculus with significant dose reduction.

In my present study I have used two separate protocol settings as above for renal calculus disease with standard dose and low dose .Under low dose protocol focused study with decreased scan length at the level of calculi was done. So, specificity and negative predictive value could not be assessed.

In the conducted study, our results demonstrated a mean average reduction of $79.92 \%$ decrease in CTDIvol in low dose CT KUB protocol when compared to standard dose CT protocol with $100 \%$ sensitivity and $100 \%$ positive predictive value in detecting the calculus and its location.

\section{Conclusion}

Low dose CT was found to be equally sensitive with equal positive predictive value in the detection and localisation of stones when compared to standard dose CT. At the same time low dose protocol helped to reduce patient radiation dose by an average of $79.92 \%$. Since urolithiasis is a recurring disease and patients are frequently subjected to repeat CT examinations, the present low dose $\mathrm{CT}$ protocol will help to reduce cumulative radiation dose to the patient without compromising the sensitivity.

\section{References}

1. Atul Sohgaura, Papiya Bigoniya. A Review on Epidemiology and Etiology of Renal Stone. American Journal of Drug Discovery and Development 2017; 7: 54-62.

2. Moira E. Dwyer, Amy E. Krambeck, Eric J. Bergstralh, Dawn S. Milliner, John C. Lieske, Andrew D. Rule .Temporal Trends in Incidence of Kidney Stones Among Children: A 25-Year Population Based Study. The Journal of Urology ; 188: 247 252.

3. Abhang Apte, Devidas Dahiphale, Anjali Pawar Dahiphale. Clinical Profile of Urolithiasis Patients at Radiodiagnosis Department of a Tertiary Care Hospital. IOSR Journal of Dental and Medical Sciences2016 ;15: 21-23.
4. Lipkin ME, Preminger GM. Imaging techniques for stone disease and methods for reducing radiation exposure. Urol Clin North Am. 2013; volume 40: 47-57.

5. Christopher L. Moore, Brock Daniels, Dinesh Singh, Seth Luty, Gowthaman Gunabushanam, Monica Ghita, et al.Ureteral Stones: Implementation of a Reduced-Dose CT Protocol in Patients in the Emergency Department with Moderate to High Likelihood of Calculi on the Basis of STONE Score.Radiology.2016;3:743-751.

6. Costello JE, Cecava ND, Tucker JE, Bau JL. $\mathrm{CT}$ radiation dose: Current controversies and dose reduction strategies. American Journal of Roentgenology. 2013; 201:1283-1290.

7. Poletti PA, Platon A, Rutschmann OT, Schmidlin FR, Iselin CE, Becker CD. Lowdose versus standard-dose CT protocol in patients with clinically suspected renal colic. American Journal of Roentgenology. 2007; 188: 927-933.

8. Sohn W, Clayman R V., Lee JY, Cohen A, Mucksavage P. Low-dose and standard computed tomography scans yield equivalent stone measurements. Urology. 2013;81:231-234.

9. Omer Mohammed Ahmed Eltayeb, Abdelazim Hussein Khalafalla, Faisal Yousif Amir and Tarig Hassan Hag Ali .Value of Hounsfield Unit in Prediction of Stone Free Rate in Management of Upper Urinary Tract Stones Using Ct and ESWL JOJ uro \& nephron. 2017; volume 2:1-4.

10. Licheng J, Yidong F, Ping W, Keqiang Y, Xueting W, Yingchen Z, et al. Unenhanced low-dose versus standard-dose CT localization in patients with upper urinary calculi for minimally invasive percutaneous nephrolithotomy (MPCNL). Indian J Med Res. 2014; volume 139: 386-392. 